\title{
Utilidad de la ecografía en el diagnóstico de los pacientes con sospecha de neoplasias del tubo digestivo
}

\author{
D. Martínez Ares, P. A. Alonso Aguirre'1, I. Martín-Granizo Barrenechea, L. Cid Gómez \\ y A. Pallarés Peral \\ Servicio de Digestivo. Complejo Hospitalario Xeral-Cíes. Complejo Hospitalario Universitario de Vigo. Pontevedra. \\ ${ }^{\prime}$ Complejo Hospitalario Juan Canalejo. A Coruña
}

\section{RESUMEN}

Introducción: el valor predictivo positivo de síntomas y signos en el diagnóstico del cáncer de colon y gástrico es bajo. Además, muchos pacientes son remitidos para descartar una neoplasia digestiva, sin síntomas que sugieran su localización, siendo el VPP de la clínica aún menor. En este trabajo evaluamos la utilidad de la ecografía como primera aproximación diagnóstica.

Material y método: se han reclutado 79 pacientes (48 varones, edad media 69,3 años). En todos ellos se realiza una ecografía antes de la endoscopia. Se evalúa la precisión en el diagnóstico del cáncer de colon y gástrico, los diagnósticos ecográficos y el número de endoscopias que se evitarían. También se buscan factores predictivos de la localización de la neoplasia.

Resultados: se han diagnosticado 5 neoplasias gástricas $(6,3 \%)$ y 12 de colon (19\%), 3 cáncer de páncreas (3,8\%), 2 neoplasias uterinas $(2,5 \%)$ y 2 hipernefromas $(2,5 \%)$. Las cifras de sensibilidad, especificidad, VPP, VPN y precisión de la ecografía fueron $80 \%$, 98,6\%; 80\%; 98,6\%; y 97,4\% respectivamente en el diagnóstico del cáncer gástrico, y del 100\%, 94,5\%; 80\%; $100 \%$ y $95,5 \%$ respectivamente en el diagnóstico del cáncer de colon. La ecografía permitió evitar el $10 \%$ de las endoscopias. El único dato que orienta la localización de la neoplasia es la presencia de anemia, que se asocia con mayor frecuencia al diagnóstico de cáncer de colon: 30,4 versus 4,3\% ( $p=0,033$ ).

Conclusiones: en los pacientes remitidos para descartar una neoplasia digestiva, con síntomas inespecíficos, se diagnostica con frecuencia de patología neoplásica ajena al tubo digestivo. Si consideramos, además, la elevada precisión diagnóstica de la ecografía, esta podría ser una muy buena primera aproximación diagnóstica.

Palabras clave: Cáncer de colon. Cáncer gástrico. Ecografía. Diagnóstico. Precisión. Utilidad.

Recibido: $15-04-08$.

Aceptado: 17-06-08

Correspondencia: David Martínez Ares. Servicio Digestivo. Hospital XeralCíes. C/ Pizarro, 22. 36204 Vigo, Pontevedra. e-mail: david.martinez. ares@sergas.es

\begin{abstract}
Background: the Positive Predictive Value (PPV) of signs and symptoms for the diagnosis of colon and gastric cancer is low. Furthermore, many patients are referred to us to discard a digestive tract neoplasm with no symptoms suggestive of its whereabouts, in whom clinical PPV is even lower. This study evaluates the usefulness of ultrasonography as a first approach to diagnosis.

Material and methods: seventy-nine patients were recruited into the study ( 48 males with an average age of 69.3 years). Ultrasonography was performed on all patients prior to endoscopy. Parameters studied included diagnostic accuracy for colon and gastric cancer, ultrasonographic diagnoses, and number of endoscopies that can be avoided. Predictive factors for neoplasm location were also studied.

Results: five gastric cancers (6.3\%), 12 colon cancers (15,1\%), 3 pancreatic cancers (3.8\%), 2 uterine neoplasms (2.5\%), and 2 hypernephromas (2.5\%) were diagnosed. The figures for sensitivity, specificity, PPV, Negative Predictive Value (NPV) and global accuracy of ultrasonography were $80 \%, 98.6 \%, 80 \%, 98.6 \%$, and $97.4 \%$, respectively, for gastric cancer, while these figures were 100\%, $94.5 \%, 80 \%, 100 \%$, and $95.5 \%$, respectively, for colon cancer. Ultrasonography enabled to avoid $10 \%$ of endoscopic explorations. The only parameter that helps locate a neoplasm is the presence of anemia, which is more frequently associated with a diagnosis of colon cancer: 30.4 versus $4.3 \%(p=0.033)$.

Conclusions: in patients without specific symptoms who were sent to us for discarding digestive tract neoplasm, "extra-digestive" neoplasms were frequently diagnosed. If we further take into account the high diagnostic accuracy of ultrasonography, then this procedure could be a very good first approach towards such diagnosis
\end{abstract}

Key words: Colon cancer. Gastric cancer. Ultrasonography. Diagnosis. Accuracy. Usefulness.

Martínez Ares D, Alonso Aguirre PA, Martín-Granizo Barrenechea I, Cid Gómez L, Pallarés Peral A. Utilidad de la ecografía en el diagnóstico de los pacientes con sospecha de neoplasias del tubo digestivo. Rev Esp Enferm Dig 2008; 100: 545-551. 


\section{INTRODUCCIÓN}

Con gran frecuencia son remitidos para la realización de exploraciones endoscópicas pacientes con sospecha de patología neoplásica del tubo digestivo, pero con clínica más o menos inespecífca, del tipo de cuadros constitucionales acompañados o no de anemia ferropénica, enfermedad metastásica hepática, etc. Los pacientes serán sometidos a exploraciones endoscópicas y radiológicas del tubo digestivo, muchas de ellas no diagnósticas, pero que son molestas para los pacientes, y además representan un apreciable consumo de recursos y tampoco están exentas de riesgos.

El CCR puede presentarse clínicamente de formas muy variadas, aunque algunos signos y síntomas se pueden usar como síntomas guía en el diagnóstico, como es el caso de la alteración en el hábito intestinal y la clínica hemorrágica (1). En el cáncer de recto es frecuente la presencia de síntomas locales (tenesmo rectal, sensación de evacuación incompleta o de ocupación); por el contrario, los tumores del colon derecho son clínicamente menos expresivos y es habitual un diagnóstico más tardío en pacientes con anemia ferropénica (2). Se consideran síntomas y signos con un valor predictivo alto de CCR la rectorragia asociada a cambio del ritmo deposicional o no asociada a síntomas anales y la palpación de una masa abdominal o rectal (3); algunos autores consideran que estos signos y síntomas sólo se asocian a neoplasias avanzadas $(4,5)$ y que podrían ser relevantes únicamente en pacientes con edades superiores a 50 años (6).

El cáncer gástrico se presenta habitualmente con clínica dispéptica; no obstante, el cáncer es una de las causas más raras de dispepsia. A partir de la clínica la validez del diagnóstico de presunción para las diversas causas de dispepsia es muy baja, en torno al 55-60\%. Este porcentaje aumenta en pacientes con dispepsia funcional, ya que la prevalencia es más elevada y ello conlleva un mayor valor predictivo positivo (VPP) (alrededor del 70-75\%). Por el contrario, el VPP para la dispepsia orgánica es mucho más bajo (30\%). Por el contrario, el elevado valor predictivo negativo (VPN) para la dispepsia orgánica (90-95\%), comparado con el de la dispepsia funcional (40-45\%), permite descartar con mayor seguridad la presencia de patología orgánica (7-13).

Si el VPP bajo de la clínica nos conduce a un número elevado de exploraciones endoscópicas no diagnósticas en los pacientes en los que sospechamos un cáncer gástrico o un cáncer de colon, el número de exploraciones infructuosas en los pacientes en los que se sospecha una neoplasia, pero no somos capaces de orientar el origen más probable de la misma, será aún mayor. La frecuencia con que diagnosticaremos neoplasias gástricas o colónicas en estos pacientes será menor que cuando existe una sospecha específica y tampoco será infrecuente la presencia de patología ajena al tubo digestivo que raramente será diagnosticada mediante técnicas endoscópicas. Por ello, la búsqueda de una estrategia que permita optimizar el diagnóstico endoscópico está justificada.
La ecografía se ha mostrado sensible tanto en el diagnóstico del cáncer gástrico como en el diagnóstico del cáncer de colon, por lo que podría ser usada como primera prueba diagnóstica en estos pacientes. El objetivo de este estudio será, por lo tanto, evaluar la rentabilidad de iniciar el estudio de los pacientes con sospecha de neoplasias gastrointestinales por una ecografía: sensibilidad y especificidad de la ecografía, neoplasias extradigestivas diagnosticadas, número de exploraciones endoscópicas que se dejarían de realizar si se iniciase el proceso diagnóstico en una ecografía. Adicionalmente, como objetivo secundario, se realizará un análisis exhaustivo de las causas por las que fueron remitidos los pacientes para su estudio con objeto de determinar si existe algún dato analítico o manifestación clínica que se asocie con mayor fuerza al diagnóstico de cáncer gástrico o de cáncer colorrectal. Ello nos ayudaría a tomar decisiones en caso de un estudio ecográfico no diagnóstico o, incluso, determinar en qué pacientes el resultado de la ecografía podría resultar irrelevante.

\section{MATERIAL Y MÉTODO}

\section{Selección de pacientes}

Se han incluido en el estudio aquellos pacientes remitidos para la realización de una endoscopia digestiva con objeto de descartar la presencia de una neoplasia digestiva, pero en cuya historia clínica no aparece reflejado dato alguno que oriente en mayor medida a una neoplasia gástrica o neoplasia colónica. El reclutamiento de pacientes se realizó entre el 1 de agosto de 2004 y el 31 de diciembre de 2005. Fueron excluidos aquellos pacientes sometidos a exploraciones endoscópicas en los dos años previos o en los que no se llegase a completar el estudio endoscópico del paciente (salvo que la primera exploración realizada sea positiva, se exigen la realización de gastroscopia y colonoscopia). Dado que la ecografía no representaba la exploración para la que habían sido remitidos los pacientes, se solicitó su permiso para la realización de la técnica y su inclusión en el estudio.

En ningún caso se reevaluó clínicamente a los pacientes, usando estrictamente la información recogida en la historia clínica por el médico responsable del paciente.

\section{Metodología del estudio}

Todos los pacientes fueron sometidos a una ecografía abdominal antes de proceder a la endoscopia, lo que asegura que el ecografista permanece ciego ante el resultado de la misma. Todas las ecografías fueron realizadas por el mismo explorador, usando un ecógrafo Toshiba Nemio 10 , dotado de sonda convexa de $3,75 \mathrm{MHz}$ y lineal de 7,5 $\mathrm{MHz}$. En ningún caso se hizo uso de la repleción de la ca- 
vidad gástrica o del colon con contraste líquido, empleando siempre una técnica convencional.

Los pacientes incluidos en el estudio, como norma general, fueron remitidos en primer lugar para la realización de una gastroscopia. Ello supone un claro sesgo de selección. El estudio ecográfico, salvo en casos en los que la ecografía fue diagnóstica de patología significativa extradigestiva, no alteró el procedimiento diagnóstico iniciado en el paciente.

Se consideró la ecografía positiva al visualizar la característica imagen de engrosamiento hipoecogénico, con pérdida de la movilidad y de la estratificación de la pared del tubo digestivo, pudiendo tratarse de un engrosamiento asimétrico (Fig. 1) o circunferencial, definiendo la característica imagen denominada "pseudorriñón" o "diana” (Fig. 2).

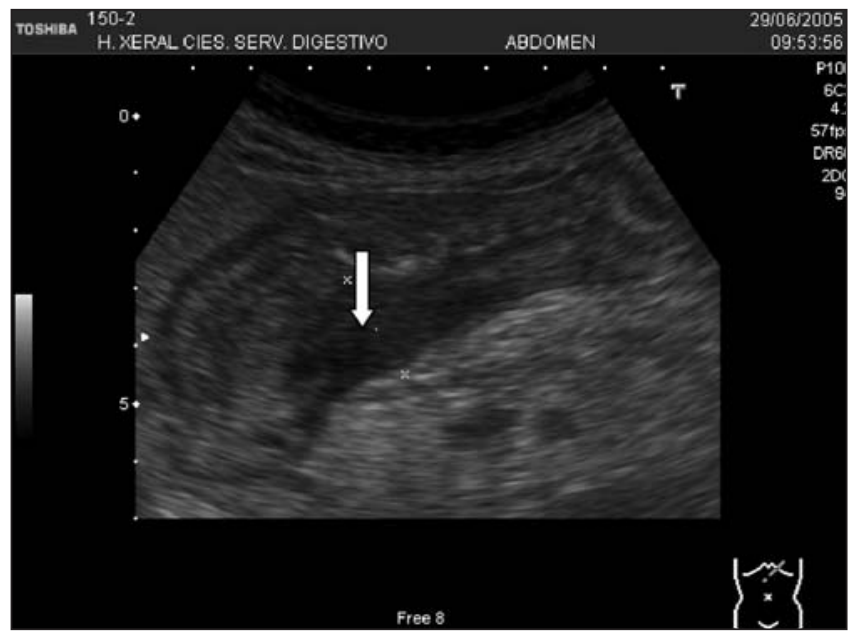

Fig. 1. Neoplasia de cara posterior de antro gástrico, que se objetiva como un engrosamiento hipoecogénico, que borra la estructura en capas y que presenta una evidente ulceración en superficie (señalado con la flecha).

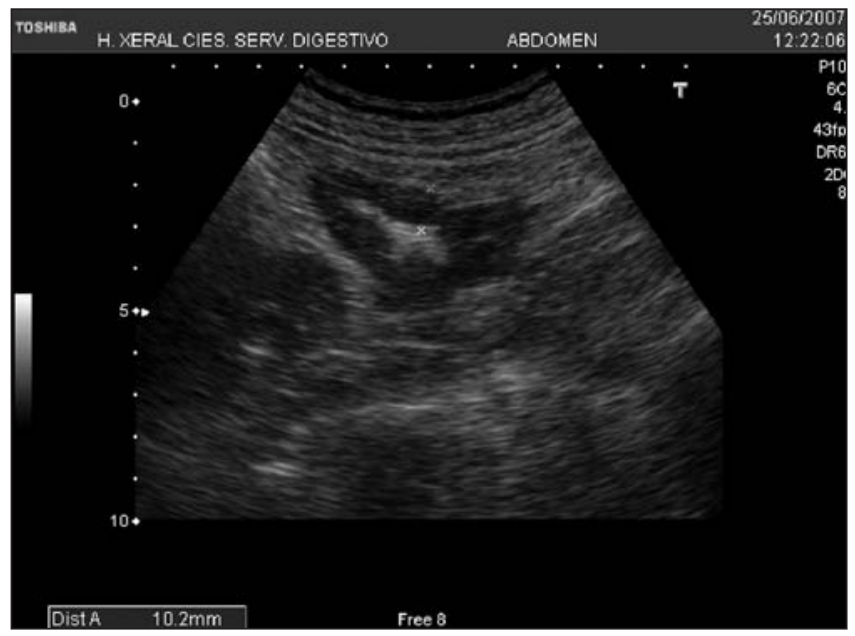

Fig. 2. Pseudorriñón típico, con un grosor parietal de 10,2 mm, con pérdida de la estructura en capas y de la movilidad, adyacente al polo superior del riñón derecho, correspondiendo a una neoplasia del ángulo hepático del colon.
El patrón oro en el diagnóstico tanto del cáncer gástrico como del cáncer de colon se consideró la endoscopia con toma de biopsias, ya que como sabemos, ninguna técnica diagnóstica ha superado su sensibilidad, y el diagnóstico histológico es obligado.

\section{Variables estudiadas}

En los pacientes incluidos en el estudio se han evaluado las características demográficas (edad y sexo), los datos clínicos e indicación del estudio, la cifra de hemoglobina y de hematocrito, el volumen corpuscular medio, los resultados de la ecografía (normal o patológica, diagnóstico de neoplasia y localización de la misma), los hallazgos endoscópicos y el diagnóstico final del paciente. Considerando la endoscopia como el patrón oro en el diagnóstico del cáncer de colon o gástrico, los hallazgos ecográficos fueron clasificados como verdaderos positivos y negativos, falsos positivos y negativos.

\section{Análisis estadístico}

El análisis estadístico se realizó mediante el software del SPSS 12.0. Se realizó la estadística descriptiva de las variables reseñadas con anterioridad. A continuación se calcularon las cifras de sensibilidad, especificidad, valores predictivos positivo (VPP) y negativo (VPN) de la ecografía en el diagnóstico de cáncer de colon y de cáncer gástrico, así como la precisión global de la técnica.

Finalmente, se realizó una búsqueda de factores asociados a un diagnóstico más probable de neoplasia gástrica o colónica. La comparación se basó en el uso de la variable Chi-cuadrado y los resultados fueron considerados estadísticamente significativos con un valor de $\mathrm{p}$ inferior a 0,05 .

\section{RESULTADOS}

Fueron valorados inicialmente para su inclusión en el estudio 90 pacientes; 11 de ellos $(12,2 \%)$ no fueron incluidos definitivamente en el análisis: 6 al no poder completarse el estudio endoscópico (esofagogastroduodenoscopia y colonoscopia hasta ciego) y 5 por una exploración ecográfica deficiente (en 2 casos las imágenes del estómago no fueron concluyentes y en 3 no se pudo explorar el colon íntegramente). El tiempo medio consumido en cada exploración ecográfica completa del abdomen fue de 13 min (rango 8-22 minutos). La edad media de los 79 pacientes incluidos en el estudio era de 69,3 años (rango de 30 a 89 años). El resto de las variables demográficas y clinicoanalíticas aparecen resumidas en la tabla I.

La ecografía fue patológica en 40 casos $(50,6 \%)$; sin embargo, tan sólo en 15 casos (19\%) la imagen era compatible con un cáncer de colon y en $5(6,3 \%)$ con una neo- 
Tabla I. Variables demográficas y clinicoanalíticas

\begin{tabular}{|c|c|}
\hline $\begin{array}{l}\text { Características de } \\
\text { los pacientes }\end{array}$ & $\begin{array}{c}\text { Número de } \\
\text { pacientes (\%) }\end{array}$ \\
\hline \multicolumn{2}{|l|}{ Edad } \\
\hline$<65$ años & 32,9 \\
\hline > 65 años & 67,1 \\
\hline \multicolumn{2}{|l|}{ Sexo } \\
\hline Varones & 60,8 \\
\hline Mujeres & 39,2 \\
\hline \multicolumn{2}{|l|}{ Manifestaciones clínicas } \\
\hline Síndrome anémico & 31,6 \\
\hline Melenas & 15,2 \\
\hline Rectorragias & 6,3 \\
\hline Vómitos en posos de café & 1,3 \\
\hline Síndrome consitucional & 17,7 \\
\hline Dolor abdominal & 16,5 \\
\hline Otras manifestaciones & 11,4 \\
\hline \multicolumn{2}{|l|}{ Hallazgos ecográficos } \\
\hline Ecografía normal & 49,4 \\
\hline Neoplasia colónica & 19 \\
\hline Neoplasia gástrica & 6,3 \\
\hline Patología no neoplásica & 12,7 \\
\hline Pancreatitis crónica & 2,5 \\
\hline Neoplasia páncreas & 3,8 \\
\hline Cáncer de útero & 2,5 \\
\hline Hipernefroma & 2,5 \\
\hline Quiste renal complicado & 1,3 \\
\hline \multicolumn{2}{|l|}{ Nivel de hematocrito } \\
\hline$<25$ & 26,6 \\
\hline $25-35$ & 38 \\
\hline$>35$ & 35,4 \\
\hline \multicolumn{2}{|l|}{ Volumen corpuscular medio } \\
\hline$<78$ & 29,1 \\
\hline$>78$ & 70,9 \\
\hline \multicolumn{2}{|l|}{ Hemoglobina ( $g / d l)$} \\
\hline$<8$ & 25,3 \\
\hline $8-11$ & 31,6 \\
\hline$>11$ & 43,1 \\
\hline
\end{tabular}

En esta tabla se resumen las variables demográficas, los parámetros analíticos, las manifestaciones clínicas y los resultados del estudio ecográfico en el grupo de pacientes que no presentaban síntomas localizadores de la neoplasia gastrointestinal.

plasia gástrica. En 10 casos $(12,7 \%)$, el estudio de la pared gástrica o del colon fue patológico, aunque sin hallazgos concluyentes de neoplasia. Por otra parte, en 2 pacientes los hallazgos ecográficos fueron sugestivos de pancreatitis crónica, y este fue el diagnóstico definitivo del paciente. Adicionalmente, el estudio ecográfico permitió el diagnóstico de 3 neoplasias pancreáticas, 2 hipernefromas, 2 neoplasias uterinas y en un paciente un quiste renal de 15 centímetros, complicado con una hemorragia intraquística.

Asumiendo el sesgo de selección que supone el que los pacientes fueran remitidos inicialmente para gastroscopia, podemos observar los siguientes resultados: fueron realizadas un total de 79 gastroscopias, diagnosticándose únicamente 5 neoplasias gástricas $(6,3 \%)$. Estos 5 pacientes y los 7 que fueron diagnosticados de neoplasias ajenas al tubo digestivo mediante la ecografía no fueron sometidos, como es lógico, a una colonoscopia. Se realizaron, por lo tanto, 67 colonoscopias, en las que se diagnosticaron 12 neoplasias de colon.

Los 12 tumores de colon fueron identificados por el ecografista (verdaderos positivos), registrándose 3 falsos positivos y ningún falso negativo. En los 52 pacientes en los que la ecografía descartó la presencia de una neoplasia de colon este resultado fue confirmado por la colonoscopia (verdaderos negativos). Estos datos se resumen en la tabla II, y de ellos se deduce que la ecografía presenta una sensibilidad del 100\%, una especificidad del 94,5\%, un VPP del $80 \%$, un VPN del $100 \%$ y una precisión del 95,5\% en el diagnóstico del cáncer de colon.

Tabla II. Resultados del diagnóstico ecográfico

\begin{tabular}{lccc}
\hline & $\begin{array}{c}\text { Pacientes } \\
\text { con CCR }\end{array}$ & $\begin{array}{c}\text { Pacientes } \\
\text { sin CCR }\end{array}$ & \\
\hline Ecografía positiva & 12 & 3 & 15 \\
Ecografía negativa & 0 & 52 & 52 \\
& 12 & 55 & 67 \\
\hline
\end{tabular}

Resumen de los resultados del diagnóstico ecográfico de CCR en pacientes con sospecha de neoplasia gastrointestinal. La sensibilidad obtenida fue del $100 \%$, una especificidad del 94,5\%, un VPP del $80 \%$, un VPN del $100 \%$ y una precisión del $95,5 \%$.

Tras la comparación del diagnóstico ecográfico y endoscópico del cáncer gástrico (Tabla III), los hallazgos ecográficos se pueden resumir en 4 verdaderos positivos, 1 falso negativo, 1 falso positivo y 73 verdaderos negativos. Ello nos permite deducir una sensibilidad del $80 \%$, una especificidad del $98,6 \%$, un VPP del $80 \%$, un VPN del $98,6 \%$ y una precisión global del $97,4 \%$.

Tabla III. Sensibilidad de la ecografía en el diagnóstico de cáncer gástrico

\begin{tabular}{lccc}
\hline & $\begin{array}{c}\text { Pacientes con } \\
\text { cáncer gástrico }\end{array}$ & $\begin{array}{c}\text { Pacientes sin } \\
\text { cáncer gástrico }\end{array}$ \\
\hline Ecografía positiva & 4 & 1 & 5 \\
Ecografía negativa & 1 & 73 & 74 \\
& 5 & 74 & 79
\end{tabular}

La sensibilidad de la ecografía en el diagnóstico de cáncer gástrico en pacientes sin síntomas específicos es del $80 \%$, una especificidad del $98,6 \%$; un VPP del $80 \%$, un VPN del $98,6 \%$ y una precisión global del $97,4 \%$

Tomando en consideración el sesgo de selección que introduce el modo en que son reclutados los pacientes y el diseño del estudio, en el que los pacientes no son remitidos inicialmente para una ecografía sino ya para una exploración endoscópica, no se puede valorar de forma ade- 
cuada el impacto real de la ecografía en el diagnóstico de estos pacientes. En cualquier caso, podemos afirmar que se han realizado en total 79 gastroscopias y 67 colonoscopias (los 5 pacientes diagnósticados de neoplasias gástricas y los 7 pacientes con neoplasias ajenas al tubo digestivo detectadas por la ecografía no fueron remitidos para colonoscopia: o se detuvo el proceso diagnóstico o se remitieron para la realización de otras técnicas de imagen). La ecografía, por lo tanto, ha permitido evitar la realización de 7 las 74 colonoscopias que se habrían de realizar tras excluir una neoplasia gástrica $(9,5 \%)$ o 7 de las 153 exploraciones endoscópicas que se habrían de realizar en total (4,5\%). No obstante, si se hubiese realizado la ecografía en primer lugar, antes de endoscopia alguna, se habrían indicado 15 colonoscopias en los pacientes con diagnóstico ecográfico de cáncer de colon, aunque en ellos se indicarían 3 colonoscopias en pacientes sin CCR (falsos positivos de la ecografía), 5 gastroscopias en los pacientes con diagnóstico ecográfico de cáncer gástrico, mientras que en los 7 pacientes con tumores malignos de otras localizaciones se evitaría la realización tanto de la gastroscopia como de la colonoscopia. En los 52 restantes pacientes, con sospecha de neoplasia, se realizarían tanto la gastroscopia como la colonoscopia. En resumen, usando la ecografía como primera aproximación diagnóstica, se habrían realizado 124 de las 153 exploraciones endoscópicas previstas si se iniciase en la gastroscopia el estudio. Es decir, se evitarían 39 exploraciones endoscópicas, lo que supone un $25,5 \%$ del total. Si añadimos las 79 ecografías, se habrían realizado un total de 203 técnicas diagnósticas. Asumiendo que en los pacientes con gastroscopia y colonoscopia negativas, pero con sospecha de neoplasia, serían sometidos a una técnica de imagen del abdomen, se realizarían 79 gastroscopias, 74 colonoscopias y al menos 62 ecografías (o TAC), lo que suma 215 exploraciones diagnósticas, 13 más que si la ecografía fuera el primer estudio diagnóstico.

Los datos clínicos y analíticos por los que fueron remitidos los pacientes se resumen en la tabla IV. De todas las variables incluidas en el análisis, probablemente por el número reducido de pacientes que fueron diagnosticados de CCR y, sobre todo, de cáncer gástrico (sólo 5 pacientes), sólo una de ellas alcanza significación estadística. No obstante el resultado puede ser trascendente, ya que la

$\begin{array}{lccc}\begin{array}{c}\text { Tabla IV. Manifestaciones clínicas y analíticas por las que } \\ \text { son remitidos los pacientes incluidos en el grupo de } \\ \text { pacientes síntomas localizadores }\end{array} \\ \begin{array}{lccc}\text { Cáncer } & \begin{array}{c}\text { Cáncer } \\ \text { gástrico (\%) }\end{array} & \text { de colon (\%) } \\ \hline \text { Variables } & 4,3 & 30,4 & 0,033 \\ & 8,0 & 22,0 & \text { NS } \\ \text { Anemia microcítica e hipocrómica } & 4,3 & 4,3 & \text { NS } \\ \text { Síndrome constitucional } & 0 & 5,3 & \text { NS } \\ \text { Hígado metastásico } & 0 & 0 & \text { NS } \\ \text { Sangrado digestivo } & 4,3 & 7,1 & \text { NS } \\ \text { Presencia de vómitos } & & & \end{array} \text { Dolor abominal } & \end{array}$

presencia de síndrome anémico, que suele traducir la presencia de una anemia microcítica e hipocrómica, es uno de los motivos de consulta más frecuentes. En efecto, en los pacientes con anemia microcítica y sospecha de neoplasia gastrointesinal, las neoplasias de colon se diagnostican con una frecuencia significativamente mayor que el cáncer gástrico: 30,4 vs. 4,3\% ( $\mathrm{p}=0,033)$.

\section{DISCUSIÓN}

El análisis de algunos de los resultados del estudio que presentamos debe ser realizado desde la perspectiva del sesgo de selección que hemos asumido desde el inicio. Este sesgo viene determinado por la forma en la que los pacientes han sido reclutados. Se trata de un estudio diseñado para evaluar la precisión de la ecografía en el diagnóstico de las neoplasias colónicas y gástricas en pacientes en los que únicamente se sospechaba la presencia de una neoplasia digestiva, sin mencionarse su localización más probable. Por tanto, no se trata de un estudio diseñado adecuadamente para evaluar el impacto de la ecografía este proceso diagnóstico. No obstante, las conclusiones que podemos extraer, si bien carecen del rigor y peso científico necesario, sí nos deben servir para incentivar estudios posteriores con un diseño adecuado.

Como ya hemos mencionado, los pacientes incluidos en el estudio habían sido remitidos todos ellos inicialmente para la realización de una gastroscopia. Esta es la norma en nuestro centro y ello se debe, probablemente, a que el clínico considera la gastroscopia menos cruenta que la colonoscopia como prueba inicial e, indudablemente, la gastroscopia es de más fácil acceso en nuestro medio, ya que la lista de espera para la colonoscopia es considerablemente mayor.

La precisión diagnóstica de la ecografía en este grupo de pacientes, tanto en el diagnóstico del cáncer de colon como del cáncer gástrico ha sido muy elevada. Tanto la sensibilidad como la especificidad alcanzadas, en ambos casos, son similares a las publicadas en la literatura, si bien es cierto que, especialmente en el caso del cáncer gástrico, el número de pacientes con este diagnóstico es muy reducido. En lo que al diagnóstico del cáncer de colon se refiere, nuestras cifras (sensibilidad del 100\%, una especificidad del 94,5\%) están bastante por encima de las obtenidas por otros autores que también han usado la ecografía convencional (14-20) y están muy próximas a las obtenidas mediante ecografía hidrocolónica (21-26). Ya en un estudio previo publicado recientemente por nuestro grupo (aunque en este caso se incluían pacientes en los que se sospechaba específicamente un cáncer de colon e incluíamos a 42 pacientes con cáncer de colon) se obtenían unas cifras de precisión diagnóstica similares (27).

En el diagnóstico del cáncer gástrico hemos obtenido una sensibilidad del $80 \%$ y una especificidad del 98,6\%; cifra muy similar a la comunicada por nosotros en pa- 
cientes en los que sí se sospechaba un cáncer gástrico y usando también ecografía convencional (28) y a la obtenida por otros autores, en su mayoría usando ecografía hidrogástrica (29-35). Como ya se ha referido, en este estudio sólo se incluyen 5 pacientes con cáncer gástrico. Aún así, las cifras no difieren sustancialmente de las publicadas con anterioridad, y alguno de los escasos estudios con los que podemos comparar nuestros resultados adolecen de las mismas limitaciones.

En definitiva, hemos obtenido unos resultados más que satisfactorios en el diagnóstico de cáncer de colon y cáncer gástrico en pacientes sin sospecha específica de estas patologías (en todos los estudios publicados hasta la fecha se han incluido pacientes con sospecha de una $\mathrm{u}$ otra patología o, en el peor de los casos, ya con un diagnóstico establecido).

Por otra parte, confirmamos lo que ya se intuía inicialmente. En efecto, la frecuencia con que diagnosticamos las neoplasias del tubo digestivo en este grupo de pacientes es muy baja. Sólo un 15,2\% de los pacientes presentaban un cáncer de colon y un 6,3\% un cáncer gástrico. Estas cifras están muy lejos del VPP del 50-60\% que parecen tener los síntomas cuando se sospecha un cáncer de colon o gástrico $(1,7)$. Adicionalmente, hemos diagnosticado neoplasias ajenas al tubo digestivo en un $8,9 \%$ (cifra superior incluso a la del cáncer gástrico). En estos pacientes, como es lógico, la práctica de una ecografía permitiría evitar la realización de exploraciones endoscópicas.

Las limitaciones referidas al diseño del estudio, como es obvio, reducen la amplitud de las conclusiones que podemos extraer, pero en un cálculo teórico basado en los resultados de precisión diagnóstica obtenidos en este estudio, observamos cómo se podría evitar la cuarta parte de las exploraciones endoscópicas realizadas en estos pacientes, no exentas de complicaciones $(36,37)$. Aunque esta afirmación tiene un sustento poco firme, parece un argumento lo suficientemente atractivo como para originar un estudio con un diseño correcto para confirmar la utilidad de la ecografía en esta tarea. Por el momento nosotros no tenemos conocimiento de que se haya sugerido esta posibilidad por otros autores, aunque nosotros ya hemos mostrado con claridad nuestra opinión (38). Esta actitud podría conducir no sólo a un "ahorro de exploraciones endoscópicas", sino también a una reducción en la demora diagnóstica en este peculiar grupo de pacientes. Como es bien conocido, el retraso en el diagnóstico de las neoplasias puede presentar repercusiones sobre el pronóstico de las misas (39).

Los pacientes portadores de neoplasias de colon derecho se presentan con frecuencia con un síndrome anémico como única manifestación (2). No obstante, en nuestra serie, sólo 1 de cada 3 pacientes que eran referidos con anemia y sospecha de neoplasia presentaban una neoplasia colónica. Cierto es que la frecuencia con que se diagnosticó un cáncer gástrico es aún menor, cifrándose en poco más de un $4 \%$. Por ello, en los pacientes con ane- mia, en caso de un estudio ecográfico no diagnóstico, podríamos sugerir la realización inicialmente de una colonoscopia. Incluso, si este hallazgo se confirmase en estudios más amplios, podríamos sugerir que en los pacientes con anemia microcítica e hipocrómica, sospechosa de originarse en una neoplasia, deberíamos iniciar el estudio por la exploración del colon, obviando el estudio ecográfico.

En definitiva, los resultados de este estudio que, como ya hemos referido, presenta innegables limitaciones, sugieren la utilidad de la ecografía como primera aproximación diagnóstica a los pacientes con sospecha de neoplasia digestiva sin síntomas localizadores, ya que diagnostican con gran precisión las lesiones colónicas y gástricas, y también detectan lesiones extraluminales. Por otra parte, parece que en los pacientes con anemia microcítica y sospecha de neoplasia, el origen más probable de la misma estará en una lesión colónica.

\section{BIBLIOGRAFÍA}

1. Grupo de trabajo de la guía de práctica clínica sobre rectorragia. Manejo del paciente con rectorragia. Guía de práctica clínica. Programa de Elaboración de Guías de Práctica Clínica en Enfermedades Digestivas, desde la Atención Primaria a la Especializada: 2. Barcelona: Asociación Española de Gastroenterología, Sociedad Española de Medicina de Familia y Comunitaria y Centro Cochrane Iberoamericano; 2002.

2. Stellon AJ, Kenwright SE. Iron deficiency anaemia in general practice: presentations and investigations. Br J Clin Pract 1997; 51: 78-80.

3. Scottish Intercollegiate Guidelines Network (SIGN). Management of colorectal cancer, 2003. Disponible en: http://www.sign.ac.uk/pdf/ sign67.pdf

4. Helfand M, Marton KI, Zimmer-Gembeck MJ, Sox HC Jr. History of visible rectal bleeding in a primary care population. Initial assessment and 10-year follow-up. JAMA 1997; 277: 44-8.

5. Eckardt VF, Schmitt T, Kanzler G, Eckardt AJ, Bernhard G. Does scant hematochezia necessitate the performance of total colonoscopy? Endoscopy 2002; 34: 599-603.

6. Winawer S, Fletcher R, Rex D, Bond J, Burt R, Ferrucci J, et al. Colorectal cancer screening and surveillance: clinical guidelines and rationale-update based on new evidence. Gastroenterology 2003; 124: 544-60.

7. Johannessen T, Petersen H, Kleveland PM, Dybdahl JH, Sandvik AK, Brenna E, et al. The predictive value of history in dyspepsia. Scand J Gastroenterol 1990; 25: 689-97.

8. Muris JW, Starmans R, Pop P, Crebolder HF, Knottnerus JA. Discriminant value of symptoms in patients with dyspepsia. J Fam Pract 1994; 38: 139-43.

9. Bytzer P, Hansen JM, Havelund T, Malchow-Moller A, Schaffalitzky de Muckadell OB. Predicting endoscopic diagnosis in the dyspeptic patient: the value of clinical judgement. Eur J Gastroenterol Hepatol 1996; 8: 359-63.

10. Hansen JM, Bytzer P, Schaffalitzky De Muckadell OB. Management of dyspeptic patients in primary care. Value of the unaided clinical diagnosis and of dyspepsia subgrouping. Scand J Gastroenterol 1998; 33: 799-805.

11. Bustamante M, Ferrando MJ, Devesa F, Borghol A. Predicción del diagnóstico endoscópico en el paciente con dispepsia: valor del síntoma predominante de presentación y de la presunción clínica inicial. Gastroenterol Hepatol 2000; 23: 66-70.

12. Heikkinen M, Pikkarainen P, Eskelinen M, Julkunen R. GPs' ability to diagnose dyspepsia based only on physical examination and patient history. Scand J Prim Health Care 2000; 18: 99-104.

13. Danish dyspepsia study group. Value of the unaided clinical diagnosis in dyspeptic patients in primary care. Am J Gastroenterol 2001; 96: 1417-21. 
14. Rutgeerts LJ, Verbanck JJ, Crape AW, Buyse BM, Ghillebert GL. Detection of colorectal cancer by routine ultrasound. J Belge Radiol 1991; 74: 11-3.

15. Uchida M, Sakoda J, Fujitoh H, Kumabe T, Oshibuchi M, Hayabuchi $\mathrm{N}$, et al. Reappraisal of the clinical usefulness of transabdominal ultrasonography for advanced colon cancer--a study of tumor detection. Nippon Igaku Hoshasen Gakkai Zasshi 1993; 53: 261-5.

16. Richardson NG, Heriot AG, Kumar D, Joseph AE. Abdominal ultrasonography in the diagnosis of colonic cancer. Br J Surg 1998; 85: 530-3.

17. Loftus WK, Metreweli C, Sung JJY, Yang WT, Leung VKS, Set PAK. Ultrasound, CT and colonoscopy of colonic cancer. B J Radiol 1999; 72: 144-8

18. Lim JH, Ko YT, Lee DH, Lee HW, Lim JW. Determining the site and causes of colonic obstruction with sonography. AJR Am J Roentgenol 1994; 163: 1113-7.

19. Grunshaw ND, Renwick IG, Scarisbrick G, Nasmyth DG. Prospective evaluation of ultrasound in distal ileal and colonic obstruction. Clin Radiol 2000; 55: 356-62.

20. Shirahama M, Koga T, Ishibashi H, Uchida S, Ohta Y. Sonographic features of colon carcinoma seen with high-frequency transabdominal ultrasound. J Clin Ultrasound 1994; 22: 359-65.

21. Limberg B. Diagnosis and staging of colonic tumors by conventional abdominal sonography as compared with hydrocolonic sonography. N Engl J Med 1992; 327: 65-9.

22. Hernández-Socorro CR, Guerra C, Hernández-Romero J, Rey A, López-Facal P, Álvarez-Santullano V. Colorectal carcinomas: diagnosis and preoperative staging by hydrocolonic sonography. Surgery 1995; 117: 609-15.

23. Segura JM, Molina E, Herrera A, Berges MA, Erdozain JC, Arjonilla A, et al. Hidrocolonic ultrasonography in the detection of tumoral processes in the inferior gastrointestinal tract. Rev Esp Enferm Dig 1998; 90: 779-87.

24. Walter DF, Govil S, William RR, Bhargava N, Chandy G. Colonic sonography: preliminary observations. Clin Radiol 1993; 47: 200-4.

25. Dux M, Richter GM, Roeren T, Heuschen U, Kauffmann GW. Gastrointestinal imaging with hydrosonography and hydro-CT. Rofo 1996; 164: 359-67.

26. Candia C, Ciacci V, Di Segni R, Santini E. Hydrocolonic sonography in the study of colonic diseases. Comparison with double-contrast enema. Radiol Med (Torino) 1995; 89: 258-63.
27. Martínez-Ares D, Martín-Granizo Barrenechea I, Souto-Ruzo J, Yánez López J, Pallares Peral A, Vázquez-Iglesias JL. The value of abdominal ultrasound in the diagnosis of colon cancer. Rev Esp Enferm Dig 2005; 97: 877-86.

28. Martínez D, Martín Granizo I, Rodríguez JI, Martínez J, Rodríguez D, Pereira S, et al. Diagnóstico ecográfico del cáncer gástrico en pacientes sintomáticos. Rev Esp Enferm Dig 2005 (Sup II): 22.

29. Worlicek H, Dunz D, Engelhard K. Ultrasonic examination of the wall of the fluid-filled stomach. J Clin Ultrasound 1989; 17: 5-14.

30. Boyacioglu S, Dolar E, Acar Y, Dalay R, Temucin G. Ultrasonographic scoring system: an auxiliary to differential diagnosis of gastric pathologies. J Clin Ultrasound 1993; 21: 97-101.

31. Tous F, Busto M. Assessment of abdominal sonography in the diagnosis of tumors of the gastroduodenal tract. J Clin Ultrasound 1997 25: 243-7.

32. Segura Cabral JM, Conde Gacho P, Comas Redondo C, Olveira Martín A, Segura Grau A, Erdozain Sosa JC, et al. The usefulness of hydrogastric ultrasonography in the detection of neoplastic lesions of the gastric wall. Gastroenterol Hepatol 1999; 22: 63-6.

33. Quiroz Moreno R, Morales Guzman MI, Cervantes Monroy JA, Rueda Torre G, Díaz Caldelas L. Exactness of transcutaneous sonography in the diagnosis of gastric wall lesions. Rev Gastroentero Mex 2003; 68: 245-52.

34. Lim JH, Ko YT, Lee DH. Transabdominal US staging of gastric cancer. Abdom Imaging 1994; 19: 527-31.

35. Richter GM, Dux M, Roeren T, Heuschen U, Kauffmann GW. Gastrointestinal diagnosis with hydrosonography and hydro-CT. 1: stomach carcinoma. Rofo 1996; 164: 281-9.

36. García Martínez MT, Ruano Poblador A, Galán Raposo L, Gay Fernández AM, Casal Núñez JE. Perforation after colonoscopy: our 16year experience. Rev Esp Enferm Dig 2007; 99: 588-92.

37. Tulchinsky H, Madhala-Givon O, Wasserberg N, Lelcuk S, Niv Y. Incidence and management of colonoscopic perforations: 8 years' experience. World J Gastroenterol 2006; 14 (12): 4211-3.

38. Martínez Ares D, Martín-Granizo Barrenechea I, Pallarés Peral A. Ecografía percutánea en el cáncer de colon. Rev Esp Enferm Dig 2007; 99 (Supl II): 59-62.

39. Gómez-Domínguez E, Trapero-Marugán M, del Pozo AJ, Cantero J, Gisbert JP, Maté J. The colorectal carcinoma prognosis factors. Significance of diagnosis delay. Rev Esp Enferm Dig 2006; 98: $322-9$. 